\title{
A Simulation Model of Hall Sensor Misalignment in BLDC Motors
}

\author{
Chung-Wen Hung \\ Department of Electrical Engineering, National Yunlin University of Science \& Technology, \\ 123, Sec. 3, University Road, Douliou, Yunlin, 64002, Taiwan \\ Chu-Lin Hsu \\ Department of Electrical Engineering, National Yunlin University of Science \& Technology, \\ 123, Sec. 3, University Road, Douliou, Yunlin, 64002, Taiwan \\ E-mail:wenhung@yuntech.edu.tw,B10100061@yuntech.edu.tw \\ www.yuntech.edu.tw
}

\begin{abstract}
A model for simulating the output signals of Hall sensors installed with misalignment in a brushless DC (BLDC) motor is proposed in this paper. In the past, Hall sensors have been very widely adopted to evaluate the commutation timing and rotation speed of a BLDC motor. However, no simulation model is yet available to take into account the misalignment of Hall sensors in order to obtain correct sensor output signals for further post processing. How the model is developed will be discussed in detail, and the proposed model will be carefully validated as well.
\end{abstract}

Keywords: simulation model, BLDC motor, Hall sensor, misalignment.

\section{Introduction}

Brushless DC (BLDC) motors are popularly used nowadays due to its high power density, low maintenance requirement, and easy control. Most BLDC motors are equipped with Hall sensors to evaluate the motor's commutation timing and rotational speed. However, the installed Hall sensors will more or less deviate from their desired positions in a BLDC motor. This misalignment, if not properly taken into account and compensated, will lead to torque rippling because of wrong commutation timing and incorrect speed feedback.

This phenomenon has been highlighted in the literature with possible solutions [1]-[4]. Ref. [1] proposed a Hall-effect-sensor-based position observer to mitigate the torque ripple in permanent-magnet synchronous machines, and the hardware and simulation validation showed the proposed algorithm functions well. However, this algorithm was proposed only for PMSM motors but not for BLDC motors. Ref. [2] proposed an auxiliary circuit for BLDC motors to reject the Hall sensor signal noise and capture the time interval. The circuit was implemented in a field programmable gate array (FPGA), and the experimental results showed that the circuit works as expected. This study could be a good reference for BLDC motor control integrated circuit, but the study did not discuss any simulation about Hall Sensor Misalignment. Ref. [3] focused only on the commutation behavior of the BLDC motor, and proposed a simulation model in Matlab/Simulink. Ref. [4] presented an efficient simulation model for a variable sampling system, which 
is caused by low resolution effect of Hall sensors. However, this model did not take into account the misalignment of Hall sensors in a BLDC motor.

In this paper, a model that takes into account the misalignment of BLDC motors will be discussed and presented. To carry out the simulation, the model is integrated with a full BLDC motor driver system simulation plan. Results indicate that the proposed model is reliable.

\section{The Simulation Method}

To simulate the Hall sensors' output signals in a BLDC motor, we must know the rotor's accumulated rotation phase. However, most BLDC motor simulation models output only the rotor's rotational speed. Hence, an integrator should be used to obtain the accumulated rotation phase. As shown in Fig. 1, where for simplification in explanation the number of pole pairs has been set to one, and the rotor is assumed to rotate clockwise with the three Hall sensors (i.e., Hall sensor $A, B$, and $C$, respectively, called $\mathrm{H}_{\mathrm{a}}, \mathrm{H}_{\mathrm{b}}$, and $\mathrm{H}_{\mathrm{c}}$ for brevity, in sequence in the clockwise direction) mounted 120 degrees apart on the stator. In this figure, the solid line that is diametrically opposite to the positon of $\mathrm{H}_{\mathrm{a}}$ represents the reference direction with a spatial phase of 0 degree. Hence, ideally, each position of $\mathrm{H}_{\mathrm{a}}, \mathrm{H}_{\mathrm{b}}$, and $\mathrm{H}_{\mathrm{c}}$ corresponds to a spatial phase of $\pi, 5 \pi / 3$, and $\pi / 3$, respectively. When point $\mathrm{O}$ at the rotor's pole interface rotates across the solid line or the position of $\mathrm{H}_{\mathrm{a}}$, the rotor's rotation angle is said to pass 0 or $\pi$, respectively. This will lead to a change of state in Hall sensor A's output at those two particular time instants, as illustrated in Fig. 2. If without truncation, the output of the rotational angle is monotony increasing. However, because of the cyclic-symmetric property of the rotation angle, we can always associate any rotation angle beyond $0-2 \pi$ to strictly within $0-2 \pi$. This can be done by taking a modulo-operation on the rotation angle with a divisor of $2 \pi$ and then extracting the remainder. In that case, Hall sensors A, B, and C will change their outputs when the rotation angles pass $(0$ or $\pi),(5 \pi / 3$ or $2 \pi / 3)$, and $(\pi / 3$ or $4 \pi / 3)$, respectively.

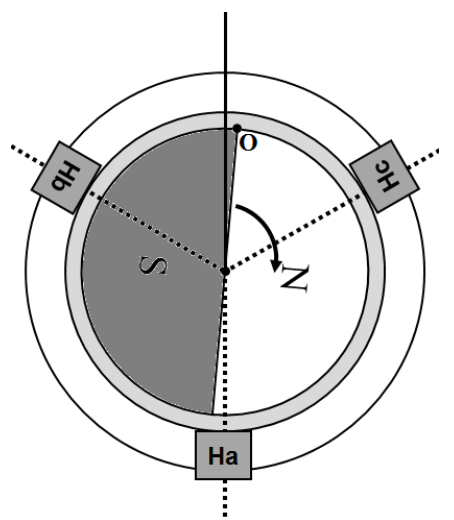

Fig. 1. Diagram of Hall-sensor and relative rotor positions

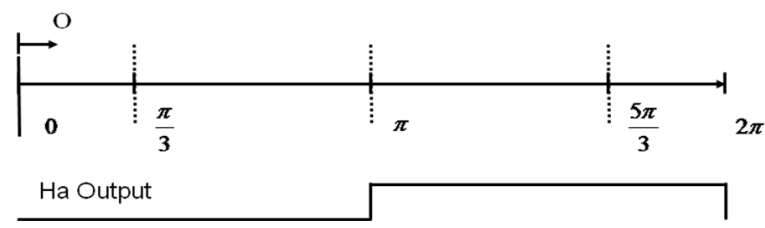

Fig. 2. The relationship between rotation angle and Hall sensor output

\section{The Proposed Simulation Model}

As discussed in the previous section, most BLDC simulation models provide only the speed output, and the rotational angle is the factor that can determine the output of a Hall sensor. An integrator can hence be adopted to integrate the rotational speed so as to obtain the rotational angle. To obtain the modulus of the rotation angle, a remainder block is needed, which comprises a divider, a rounder, and an adder. Next, the determination of the Hall sensor's output can rely on a relational operator. Fig. 3 shows the function blocks of the model for simulating the Hall sensor's output with sensor A as an example. In this figure, only one relational operator is needed, for which there are two inputs. The first input is the normalized modulus of the rotation angle (i.e., the modulus normalized with respect to $2 \pi$ ), and the second input is 0.5 , which is the normalized value of $\pi$ (still normalized with respect to $2 \pi$ ) used as a threshold in the relational operation. Similarly, the output of Hall Sensor B (C) is one value when rotational angle is greater than $5 \pi / 3(\pi / 3)$ or less than $2 \pi / 3(4 \pi / 3)$, and is the other value otherwise. 
Detailed relational operations are dictated by (1)-(3), where the symbol \% represents a modulo operation. For completeness, functional blocks for the model pertaining to the simulation of Hall sensors $\mathrm{B}$ and $\mathrm{C}$ are demonstrated in Fig. 4. Comparison of Figs. 3 and 4 reveals that two relational operators are employed for each of Hall sensors B and C, whereas only one is needed for Hall sensor A. This drawback will be eliminated in the model proposed in the next section.

\begin{tabular}{|r|l|c|}
$\operatorname{Hall}_{\mathrm{A}}(\theta)=0$, & if $(\theta \% 2 \pi)<\pi$ \\
$=1$, & if $(\theta \% 2 \pi) \square \pi$ & (1) \\
\hline $\operatorname{Hall}_{\mathrm{B}}(\theta)=1$, & if $(\theta \% 2 \pi)<2 \pi / 3$, or $(\theta \% 2 \pi) \square 5 \pi / 3$ & (2) \\
$=0$, & o. w. & \\
\hline $\operatorname{Hall}_{\mathrm{C}}(\theta)=1$, & if $(\theta \% 2 \pi) \square \pi / 3$, and $(\theta \% 2 \pi)<4 \pi / 3$ & $(3)$ \\
$=0$, & o. w.
\end{tabular}

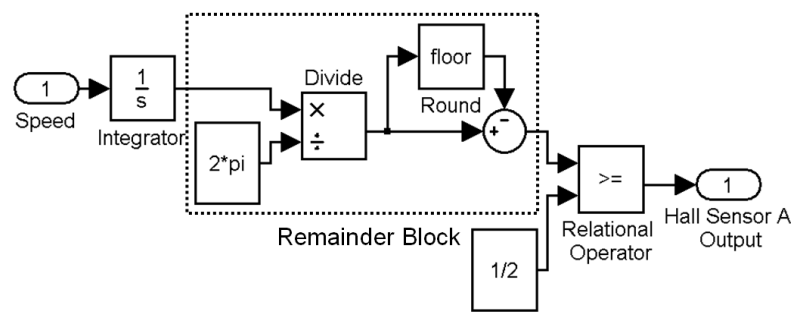

Fig. 3. Simulation model of Hall-sensor

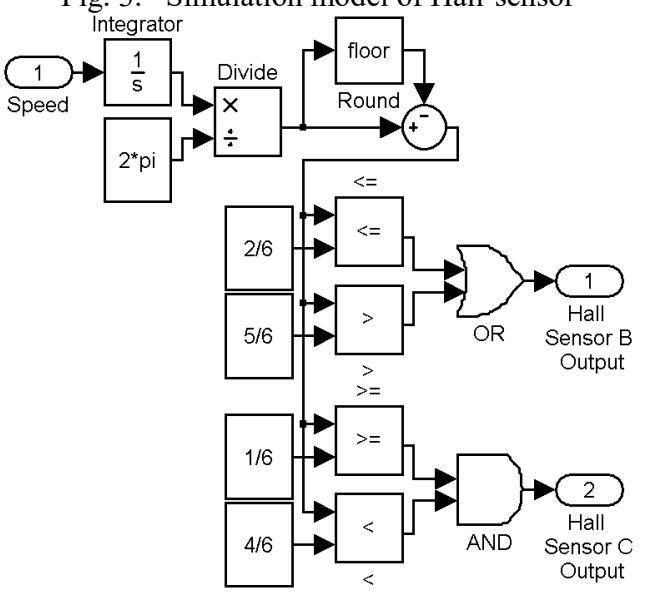

Fig. 4. Simulation model of Hall-sensor B and C

\section{The Proposed Hall Sensor Misalignment Effect Simulation}

As indicated in Figs. 3 and 4, the number of relational operators needed in the model for Hall sensor A is different from that for Hall sensors B and C. In view of the cyclic-symmetric property of the rotation angle, it is possible to implement the models such that they all need the same number of relational operators. We hence propose the equivalent one, as shown in Fig. 5, where only one relational operator is needed for each sensor output and all the three relational operators have the same threshold value of 0.5 . This simplification can be achieved because the accumulated rotational angle input to the remainder block of Hall sensor B (C) has been shifted by $4 \pi / 3(2 \pi / 3)$.

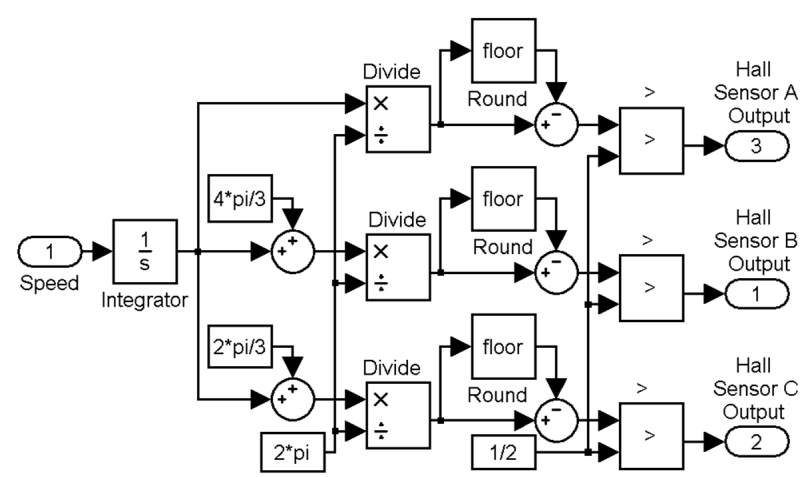

Fig. 5. Equivalent simulation model

Ideally, Hall sensors should be installed at their prescribed positions. In reality, the positions the Hall sensors installed in most BLDC motors may deviate from those prescribed ones. The simulated output signals of the misaligned Hall sensors should accurately reflect the misalignment; otherwise these simulated signals would not be useful for further processing when the development of a BLDC motor and its associated control circuit heavily rely on a complete system simulation. In Fig. 6, dot lines represent the Hall sensors' prescribed positions, and dash lines indicate the Hall sensors' actual positions. We have assumed that Hall sensor A is well aligned and that Hall sensors B and $\mathrm{C}$ deviate from their prescribed positions by $\boldsymbol{\Delta} \boldsymbol{\theta}$ and $\Delta \varphi$, respectively. The misalignment should be taken into account in the simulation that provides Hall sensors' output signals. The deviation angle $\boldsymbol{\Delta} \boldsymbol{\theta}$, when taken into account in the function blocks pertaining to Hall sensor B, leads to a new model shown in Fig. 7, where the normalized deviation angle is added to each threshold of the two relational operators. 


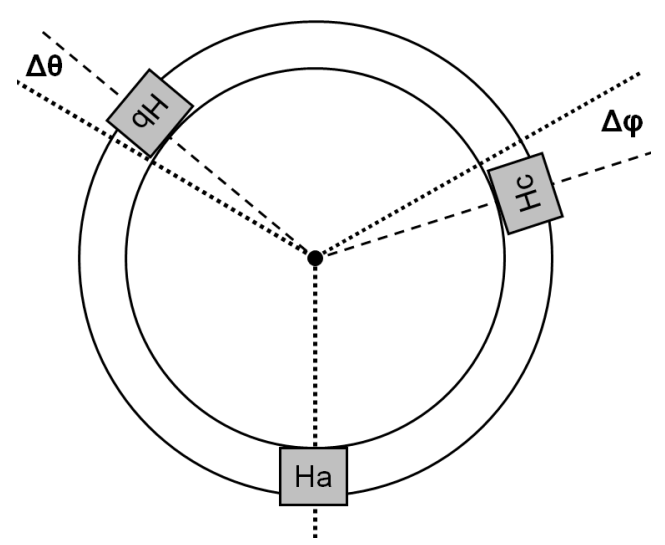

Fig. 6. Diagram of Hall-sensor with misalignment

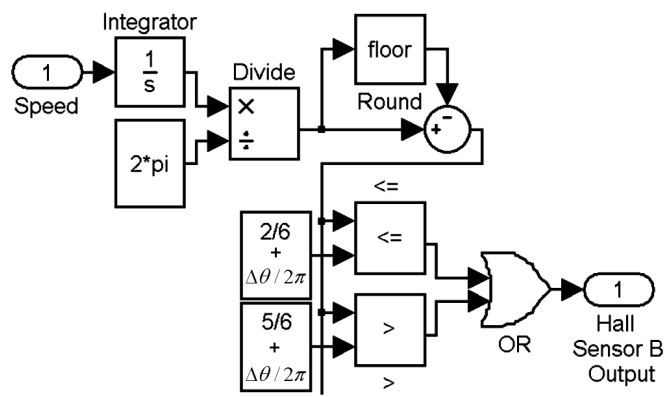

Fig.7. Misalignment simulation model of Hall-sensor B

Although intuitively correct, the model shown in Fig. 7 can still be simplified in view of the model development from Fig. 4 to Fig. 5. Because the movement between the rotor and each Hall sensor is relative, the normalized deviation angle can be subtracted from the input accumulated rotation angle. The latter choice is in fact easier to implement, and its associated model is shown in Fig. 8. So far, we have assumed that Hall sensor A is well installed without position error. However, it is clear from Fig. 8, if there is also a deviation angle for Hall sensor A, it can also be subtracted from the input accumulated rotation angle.

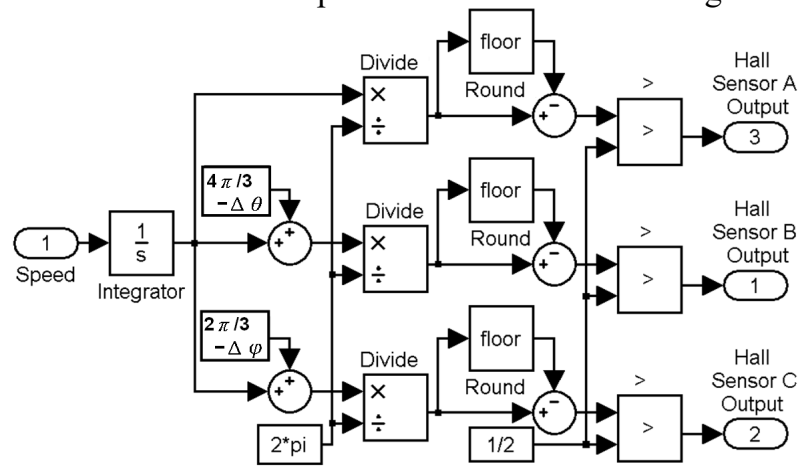

Fig. 8. Equivalent misalignment simulation model

\section{Simulation Results}

To validate the proposed Hall-sensor output model, we use as an aid a Matlab built-in model, called Brushless DC Motor Fed by Six-Step Inverter Model and to be run in the Matlab/Simulink environment. The upper half of Fig. 9 is this built-in model, and the bottom half is a duplicated one but with the original Hall-sensor output replaced by the proposed Hall-sensor output model (indicated by red circle in Fig. 9). As is understood, the input of the proposed model is the rotation speed of the rotor, and the output is connected to the same decoder in the built-in model. Fig. 10 shows the Hall sensors' output signals simulated using the upper half function blocks of Fig. 9 and those using the lower half without any misalignment. In this figure, the horizontal axis is the time in second, and the three waveforms indicate the logic-level output of $\mathrm{Ha}, \mathrm{Hb}$ and $\mathrm{Hc}$, respectively. These

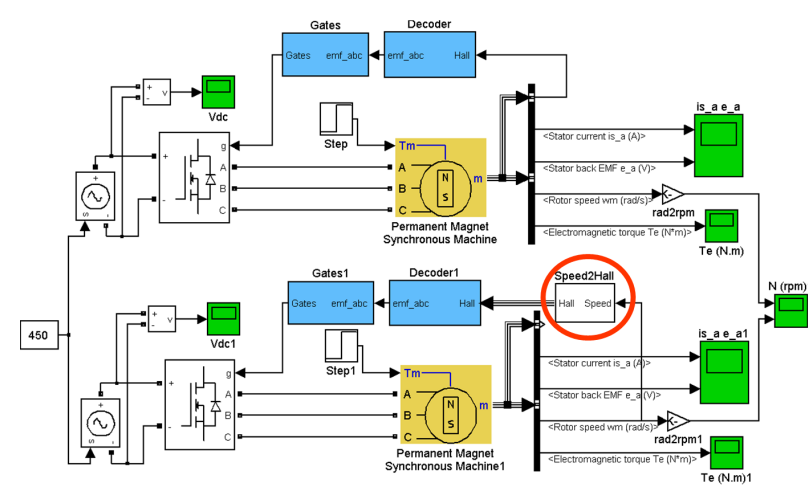

Fig. 9. The simulation of the "Brushless DC Motor Fed by Six-Step Inverter model" with misalignment simulation

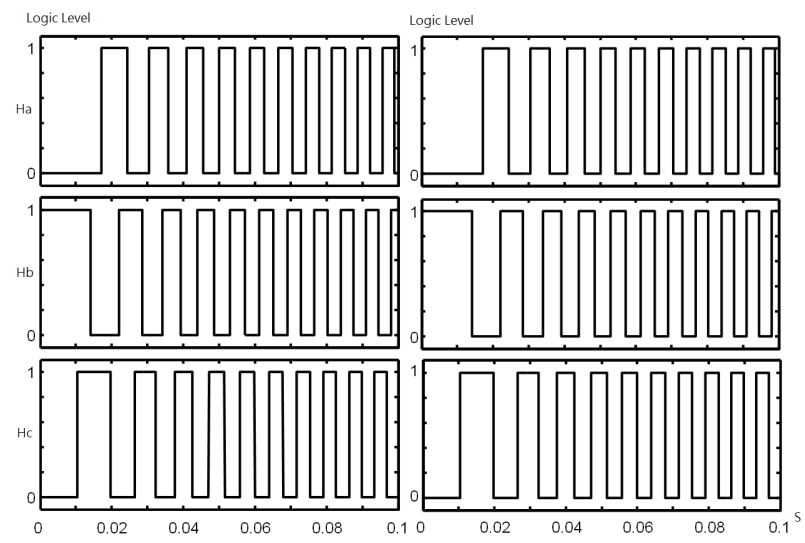

(a) Matlab Simulink demonstration (b) proposed models

Fig.10. Hall sensor signal comparison without misalignment 
two sets of results are identical, as expected. Obviously, the proposed simulation model could simulate well the Hall sensors' output without misalignment.

Next, to further validate the proposed Hall-sensor output model shown in Fig. 8, we consider the case where the misalignment is present in one of the Hall sensors, say, Hall sensor B. For that purpose, the simulation model shown in Fig. 11 is adopted, where only a motor is used for simulation. In this simulation model, the speed output is fed to the proposed Hallsensor output model with misalignment, and then the outputs of the proposed Hall-sensor output model are compared with those of the original Hall sensors. The comparison is shown in Fig. 12. Again, the horizontal axis is the time in second, and the vertical axis is the logic level of the Hall sensor's output. The solid lines in both subfigures are the outputs of Hall sensor A without misalignment, and the dash line in the upper subfigure indicates the output of Hall sensor B without misalignment as well. In contrast, the dot line in the lower subfigure shows Hall sensor B' output with a misalignment of $\boldsymbol{\Delta \theta}=\pi / 6$. When the motor runs in the steady state, meaning that the speed is uniform in time, the output pulse trains of the Hall sensors are symmetric (i.e., with a duty cycle of $50 \%$ ), whether or not with misalignment. In addition, as compared with the output of Hall sensor A, the output of Hall sensor B without misalignment is delayed by $2 \pi / 3$, whereas that of Hall sensor B with misalignment indeed shows an additional expected phase delay of $\pi / 6$. Obviously, this delay will cause wrong commutation timing and speed estimation.

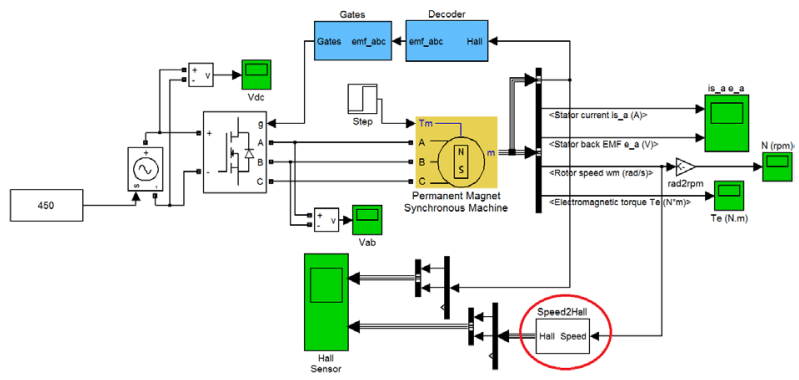

Fig. 11. The simulation of the "Brushless DC Motor Fed by Six-Step Inverter model" with the proposed Hall sensor module

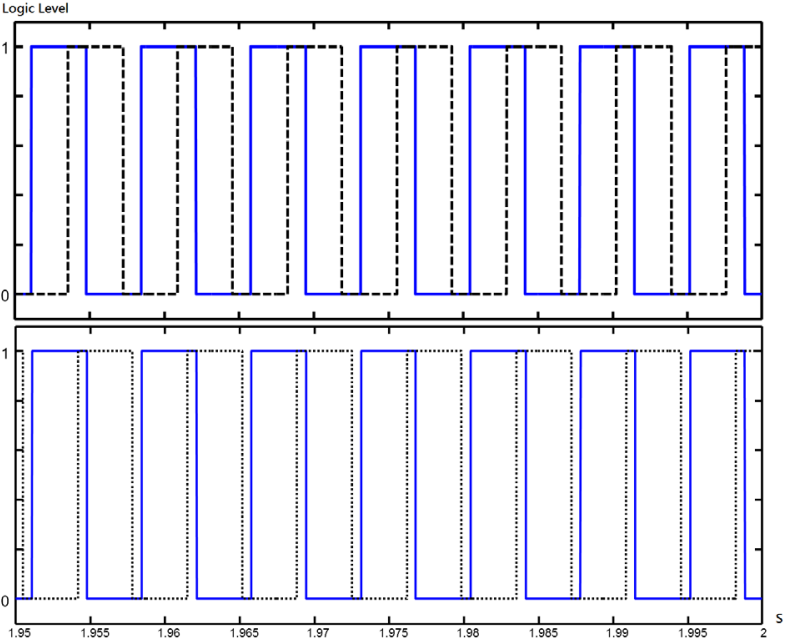

Fig. 12. Simulation result of Hall sensor B with misalignment

Finally, the speed output comparisons in Fig. 9 are shown in Fig. 13, where the dash line is the speed response of the BLDC motor without Hall sensor misalignment and the solid line presents the one with misalignment. The simulated speed response for the case without misalignment is obviously more stable than the one for the case with misalignment. In addition, there are always ripples in the speed response for the case with misalignment. However, this does not mean that the smoother response is our preferred one. This is because the simulated speed response without misalignment can also be regarded the one with the existence of the misalignment but without taking into the misalignment in the simulation. From this point of view, the simulated speed ripples that are due to the Hall sensor's misalignment and its resultant wrong rotor's commutation timing are the desired results. By appropriately compensating the misalignment effect, these ripples can be eliminated by other techniques implemented in the control circuit. If the misalignment is not taken into account in the simulation, then the ripples will not appear in simulated data and no strategies can be sought to compensate the misalignment. 


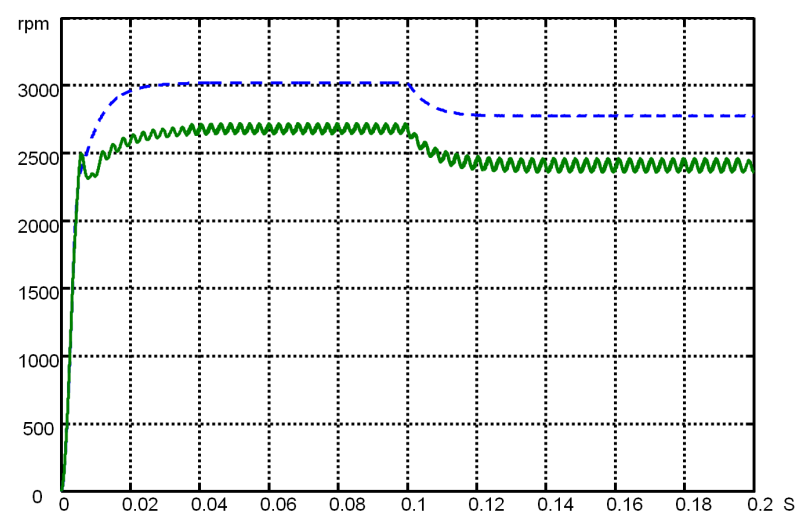

Fig. 13. Simulation result of Hall sensor B with misalignment

\section{Conclusion}

A simulation model of Hall sensor misalignment in BLDC motors is proposed in this paper. For most BLDC motors, it is very difficult and expensive to install Hall sensors in prescribed position accurately. And it will cause torque ripple and wrong speed feedback. The proposed model could simulate exactly this misalignment effect, and it is can provide a good simulation reference to engineers for designs of BLDC motor controllers.

\section{Acknowledgements}

This work is partially supported by the Ministry of Science and Technology, ROC, under contract No. NSC 104-2622-E-224-016-CC3, 104-2221-E-224-012 and MOST 105-2622-E-224-017-CC3.

\section{References}

1. P. B. Beccue, S. D. Pekarek, B. J. Deken, and A. C. Koenig, Compensation for Asymmetries and Misalignment in a Hall-Effect Position Observer Used in PMSM Torque-Ripple Control, IEEE Transactions On Industry Applications, Vol. 43, NO. 2, MARCH/APRIL 2007, pp. 560-570.

2. C.-W. Hung and J.-H. Chen, A Hall Sensor Auxiliary Circuit with Noise Rejection and Time Interval Capture Functions for BLDC Motors, Sensor Letters, Vol. 10, 2012, pp. 1178-1184.

3. B. Tibor, V. Fedák, and F. Durovský, Modeling and Simulation of the BLDC Motor in MATLAB GUI, in Proc. 2011 IEEE International Symposium on Industrial Electronics (ISIE), 27-30 June 2011 pp.1403-1407

4. C.-W. Hung, C.-T. Lin and C.-W. Liu, An Efficient Simulation Technique for the Variable Sampling Effect of BLDG Motor Applications, in Proc.33rd Annual Conference of the IEEE Industrial Electronics Society, Nov. 5-8, 2007 (Taipei, Taiwan, 2007), .pp. 1175 - 1179 